\title{
Basal Cell Adenocarcinoma of the Sublingual Gland: A Case of an Inadequate FNA Cytology Specimen Rendering Conclusive Diagnosis Difficult
}

Sohsuke Yamada ${ }^{1 *}$, Noriko Tsunenari ${ }^{2}$, Atsunori Nabeshima ${ }^{1}$, Kosho Obara ${ }^{2}$, Hirotsugu Noguchi ${ }^{1}$, Aya Nawata ${ }^{1}$, Hidetaka Uramoto ${ }^{3}$ and Toshiyuki Nakayama ${ }^{1}$

${ }^{1}$ Department of Pathology and Cell Biology, School of Medicine, University of Occupational and Environmental Health, Kitakyushu, Japan

${ }^{2}$ Department of Diagnostic Pathology, School of Medicine, University of Occupational and Environmental Health, Kitakyushu, Japan

${ }^{3}$ Department of Second Surgery, School of Medicine, University of Occupational and Environmental Health, Kitakyushu, Japan

"Corresponding author: Sohsuke Yamada, M.D., Ph.D., Department of Pathology and Cell Biology, School of Medicine, University of Occupational and Environmental Health, 1-1 Iseigaoka, Yahatanishi-ku, Kitakyushu 807-8555, Japan, Tel: 81-93-691-7426; Fax: 81-93-603-8518; E-mail: sousuke@med.uoeh-u.ac.jp

Rec date: Sep 11, 2014, Acc date: Sep 26, 2014, Pub date: Sep 28, 2014

Copyright: @ 2014 Yamada S, et al. This is an open-access article distributed under the terms of the Creative Commons Attribution License, which permits unrestricted use, distribution, and reproduction in any medium, provided the original author and source are credited.

\begin{abstract}
A history of a gradual increase in swelling mass was presented in the sublingual gland of a 72-year-old female. The inadequate cytologic specimens retrospectively contained some clusters of three-dimensional monomorphic and round basaloid cells having hyperchromatic small nuclei and scant cytoplasm, along with a small amount of spherical globules of amorphous material and myxoid stroma, adjacent to few squamous metaplastic tumor nests. We first interpreted it as a pleomorphic adenoma, basal cell adenoma (BCA), or adenoid cystic carcinoma (ACC). A tumor extirpation was performed, and gross examination revealed a non-capsulated and ill-defined tumor lesion, looking grayish to yellowish-white, focally associated with fat invasion. On microscopic examination, the tumor was predominantly composed of a proliferation of small to medium-sized mildly atypical epithelial cells having very rare mitoses, often arranged in a cribriform or alveolar growth pattern with peripheral nuclear palisading and scattered squamous differentiation, embedded in a prominent eosinophilic hyaline material. Therefore, we finally made a diagnosis of invasive basal cell adenocarcinoma (BCAC), defined as the malignant counterpart of benign BCA. We should be aware that owing to its characteristic features, cytopathologists might be able to determine correct diagnosis, based on multiple and adequate samplings.
\end{abstract}

Keywords: Basal cell adenocarcinoma (BCAC); Sublingual gland; Cytopathology; Basal cell adenoma (BCA)

\section{Introduction}

Among all epithelial salivary gland neoplasms, basal cell adenocarcinoma (BCAC) is uncommon and accounts for approximately 1 to $2 \%$, whereas constitutes up to nearly $3 \%$ of the epithelial malignancies [1]. BCAC of the salivary gland is defined as the malignant counterpart of benign basal cell adenoma (BCA) $[1,2]$, and often poses a diagnostic challenge to clinicians and cytopathologists, since its entity is much more difficult to diagnose pre-operatively on an inadequate and small sample. Actually, the WHO classification in 2005 simply defined BCAC as an infiltrative epithelial neoplasm, morphologically and cytopathologically similar to BCA in part [2]. Moreover, BCACs can be asymptomatic as most of them are not widely invasive on gross findings and often have similar clinical presentations as $\mathrm{BCA}$, however, patients with BCAC tend to recur and only occasionally metastasize due to infiltrative and locally destructive behavior [1-3]. By contrast, the cytomorpholology including histopathological cribriform or solid growth patterns of BCAC can result in the misdiagnosis of adenoid cystic carcinoma (ACC), which is much more aggressive than not only BCA but BCAC [2-4]. Thus, early accurate diagnosis and following surgical treatment of BCACs can allow for an improved quality of life and likely increase their survival rates [4]. However, there have been very few available papers describing the cytological features of BCAC and/or BCA from fine needle aspiration (FNA) specimens. Herein we reported a rare case of BCAC originated from the sublingual gland, rendering conclusive diagnosis difficult on an inadequate FNA cytology specimen.

\section{Case Report}

The patient presented here, a 72-year-old female with an unremarkable previous medical history, had a complaint of gradual increase in swelling mass of the right oral floor, covered by smooth surface of oral mucosa (Figure 1A). Laboratory data, including blood cell count, chemistry and tumor markers, were within normal limits. A neck CT scan revealed a heterogeneously but mildly enhanced and relatively well-demarcated nodule, measuring approximately $25 \times 15$ $\mathrm{mm}$ in diameter, arising possibly from the sublingual gland and attached to the right inner side of mandible bone (Figure 1A). Fullbody CT scans disclosed no definite evidence of metastases in the lymph nodes or other organs. The inadequate specimen from the FNA cytology retrospectively contained some clusters of three-dimensional monomorphic and round basaloid cells having hyperchromatic small nuclei and scant cytoplasm, along with a small amount of spherical globules of amorphous material and myxoid stroma, adjacent to few squamous metaplastic tumor nests, without any evidence of necrotic or hemorrhagic backgrounds (Figure 1B). Clusters of tumor cells sometimes and likely exhibited peripheral palisading of nuclei around the spherical globules of material (Figure 1B). Due to the inadequateness of cytologic specimens, Papanicolaou stains were only performed, but Giemsa staining was not. We first interpreted it to be eithera pleomorphic adenoma, BCA, or ACC, because its cytological features overlapped with those of each tumor. A tumor extirpation with combined partial resection of adjacent sublingual gland was thus 
Citation: Yamada S, Tsunenari N, Nabeshima A, Obara K, Noguchi H, et al. (2014) Basal Cell Adenocarcinoma of the Sublingual Gland: A Case of an Inadequate FNA Cytology Specimen Rendering Conclusive Diagnosis Difficult. J Cytol Histol 5: 281. doi: $10.4172 / 2157-7099.1000281$

Page 2 of 3

performed, and gross examination displayed a relatively firm, noncapsulated and ill-defined nodular lesion, looking grayish to yellowishwhite in color (Figure 1C). On scanning magnification, the tumor showed a poorly-circumscribed and uncapsulated nodule, involving the salivary gland and focally invading the surrounding fat (Figure 1D). Resection was diagnosed as most likely complete by this histopathological examination. Microscopically, the tumor was predominantly composed of a proliferation of mildly atypical epithelial cells, often arranged in a cribriform (Figure 2A, B) or alveolar (Figure 2C) growth pattern with peripheral nuclear palisading (Figure 2A) and sometimes squamous differentiation (Figure 2C), embedded in a prominent eosinophilic hyaline material (Figure 2A). On high-power view, these neoplastic cells revealed a small to medium-sized and round shape, having hyperchromatic enlarged nuclei, occasionally conspicuous nucleoli, and scant cytoplasm (Figure $2 \mathrm{~B}, \mathrm{C}$ ). Mitotic figures were very rarely encountered. Moreover, these tumor nests partly revealed not only fat invasion but perineural involvement (Figure 2D). Immunohistochemical findings with S-100 protein (S-100 A and B; Dako Cytomation Co., Glostrup, Denmark, diluted 1:900) highlighted the latter (Figure 2D). Immunohistochemically, the inner cells of tumor nests were positive for cytokeratins (AE1/AE3; CHEMICON International, Inc., Temecula, California, USA, diluted 1:200, and 34ßE12; Leica Microsystems, Wetzlar, Germany, diluted 1:200), whereas the outer cells of them were positive for $\alpha$-smooth muscle actin ( $\alpha$-SMA; Dako, diluted 1:150) and p63 (Dako, diluted 1:30). On the other hand, Ki67 (MIB-1; Dako, diluted 1:50) labeling index was less than $2 \%$ in the proliferating mildly atypical cells of the tumor nests. Based on all these features, we finally made a diagnosis of invasive BCAC arising from the sublingual gland. There were no overt benign pleomorphic adenoma components, within our thorough investigation. To date, approximately 4 years routine follow-up after the surgery is established, and the patient remains well and neither recurrences nor metastases have been identified.

\section{Discussion}

The overall nature of BCACs as well as BCAs from the salivary glands appears generally indolent, whereas aggressive clinical treatment in the early stage for ACCs should be only the hope for better prognosis, due to a higher-grade, infiltrative and destructive malignant tumor [1-4]. Thus, it is critical to establish an accurate preoperative diagnosis by FNA cytology, the clinical utility of which in diagnosing salivary gland tumors has been generalized. However, cytological findings for the BCACs and BCAs have been rarely described more in detail in the literatures, since the category of BCAC can particularly be indistinct, questioned and might have a critical flaw. In fact, BCAC with extensive involvement of skeletal muscle and/or skin is rarely encountered, but BCA might also be considered as an infiltrative neoplasm [1-3]. Therefore, those entities are much more difficult to diagnose pre-operatively. To the best of our knowledge, this is actually the second single-case report of BCAC from the sublingual gland [5], and the first report of sublingual BCAC, especially focusing on its cytomorphologic findings. Its cytologic features could reflect the histopathological ones, but nearly identical to those of BCA, showing clusters of small and blue basaloid cells having hyperchromatic nuclei and scant cytoplasm, accompanied by a palisading or rosette-like pattern with a substantial amount of central spherical globules of amorphous material, often without any evidence of the necrotic or hemorrhagic backgrounds $[1,6,7]$. As in the present case, while the specimens were inadequate, the cytologic features were almost similar to those as described above, even though rare squamous metaplastic tumor nests were seen. ACC is another most important differential diagnosis, and its cytology findings reveal that the nuclei of ACC cells are less regular with a coarsely granular chromatin, more prominent nucleoli and a larger number of mitotic figures [4,7]. Moreover, nuclear molding is a common feature of ACC, whereas squamous metaplasia is not at all, contrary to that of BCAC $[1,2,4,7]$. Despite that, a confident and accurate diagnosis of BCAC after distinguishing from BCA should be impossible merely on cytology, due to cytomorphologic variety, sampling errors, lack of experience, and/or misinterpretation [1,7]. Therefore, in any cases with a strong clinical suspicion of malignant salivary gland tumors, multiple and CT-guided, if possible, FNA cytology should be performed and its suspicion must be raised to alert the cytopathologists, at the very least.

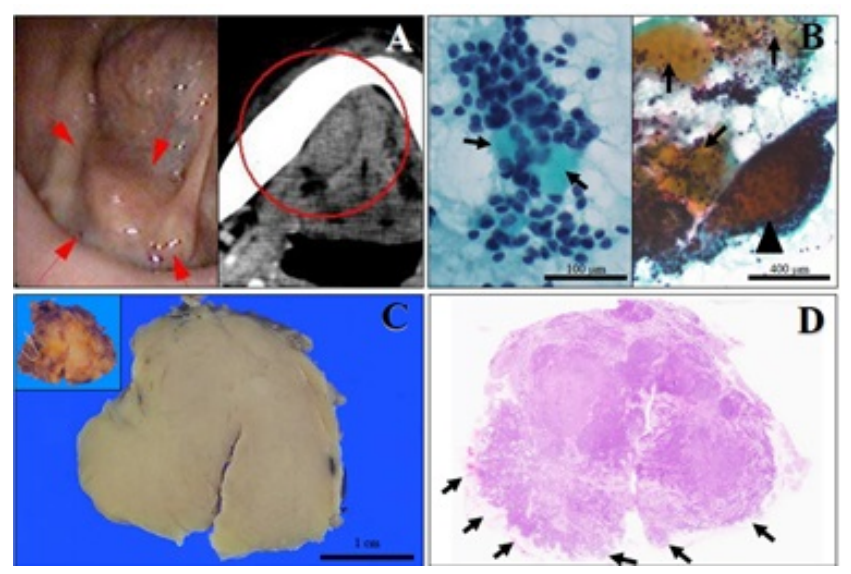

Figure 1: The findings of neck CT at surgery, FNA cytomorphologic, gross and microscopic examinations of the invasive BCAC specimens. (A) The patient had a complaint of gradual increase in swelling mass (arrows) of the right oral floor, covered by smooth surface of oral mucosa (left). A neck CT scan showed a heterogeneously but mildly enhanced and relatively welldemarcated nodule (in circle), measuring approximately $25 \times 15$ $\mathrm{mm}$ in diameter, arising possibly from the sublingual gland and attached to the right inner side of mandible bone (right). (B) The inadequate cytology specimen (Papanicolaou stains) retrospectively contained some clusters of three-dimensional monomorphic and round basaloid cells having hyperchromatic small nuclei and scant cytoplasm (left), along with a small amount of spherical globules of amorphous material (arrows, left) and myxoid stroma (arrows, right), adjacent to few squamous metaplastic tumor nests (arrowhead, right), without any evidence of necrotic or hemorrhagic backgrounds. Clusters of tumor cells sometimes and likely exhibited peripheral palisading of nuclei around the spherical globules of material (left). Bars $=100 \mu \mathrm{m}$ (left) and $400 \mu \mathrm{m}$ (right). (C) A tumor extirpation with combined partial resection of adjacent sublingual gland was performed (inset). Cut surface displayed a relatively firm, non-capsulated and ill-defined nodular lesion, looking grayish to yellowish-white in color. Bar $=1 \mathrm{~cm}$. (D) On scanning magnification, the tumor showed a poorlycircumscribed and uncapsulated nodule, involving the salivary gland and focally invading the surrounding fat (arrows) (H\&E stains). 
Citation: Yamada S, Tsunenari N, Nabeshima A, Obara K, Noguchi H, et al. (2014) Basal Cell Adenocarcinoma of the Sublingual Gland: A Case of an Inadequate FNA Cytology Specimen Rendering Conclusive Diagnosis Difficult. J Cytol Histol 5: 281. doi: $10.4172 / 2157-7099.1000281$

Page 3 of 3

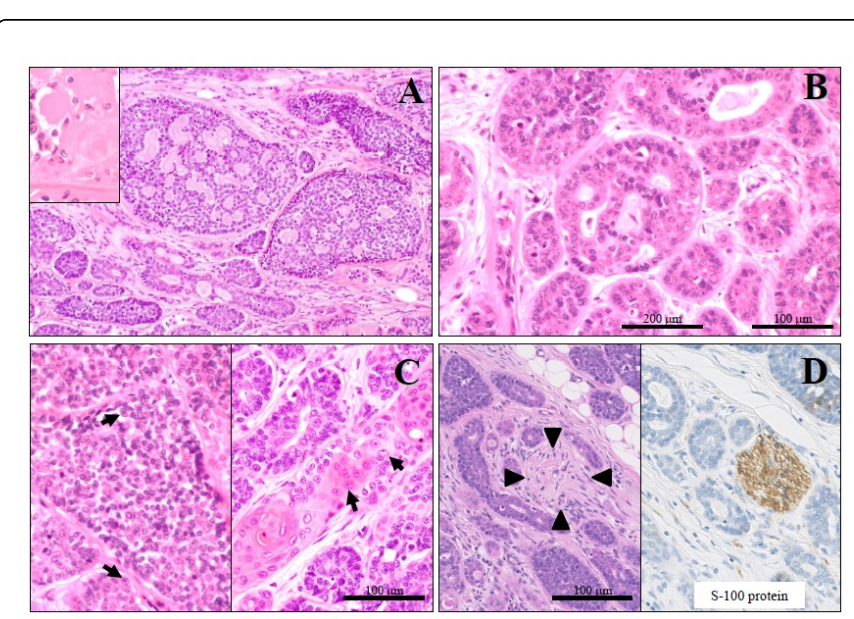

Figure 2: Microscopic examinations of the sublingual BCAC. (A) (B)(C) The tumor was predominantly composed of a proliferation of mildly atypical epithelial cells, often arranged in a cribriform (A, $B)$ or alveolar (C, left) growth pattern with peripheral nuclear palisading $(\mathrm{A})$ and sometimes squamous differentiation $(\mathrm{C}$, right), embedded in a prominent eosinophilic hyaline material (A, inset). On high-power view, these neoplastic cells revealed a small to medium-sized and round shape, having hyperchromatic enlarged nuclei, occasionally conspicuous nucleoli (arrows), and scant cytoplasm (B, C). Mitotic figures were very rarely encountered ( $\mathrm{H} \& \mathrm{E}$ stains). Bars $=200 \mu \mathrm{m}(\mathrm{A})$ and $100 \mu \mathrm{m}(\mathrm{B}, \mathrm{C})$. (D) In addition, these tumor nests showed perineural involvement (arrowheads) (H\&E stains) (left). Immunohistochemistry for S-100 protein highlighted it (right). Bar $=100 \mu \mathrm{m}$.

Furthermore, according to more recent research paper, an immunohistochemical analysis indicates that $\beta$-catenin is highly and specifically expressed in patients with BCACs and BCAs [8], but S-100P protein is almost not, unlike in those with ACCs. Therefore, we can propose that immunostaining for those 2 markers together with MIB-1 labeling indices on cytologic smears or cell block preparations might be very useful for the exclusion from ACC, at least.
We herein reported a case of a BCAC arising from the sublingual gland, tentatively misdiagnosed as the suspicion of ACC, on the examination of the inadequate cytology. All cytopathologists should be aware that its clinicopathologically characteristic features, as well as multiple and adequate FNA specimens, might lead to a correct diagnosis. Nevertheless, future cytomoprphological studies will be further required to determine whether only cytology specimens can distinguish $\mathrm{BCAC}$ from BCA after collecting and examining a larger number of those cases.

\section{References}

1. Ellis GL, Auclair PL (2008) Basal Cell Adenocarcinoma. In: Tumors of the Salivary Glands. Atlas of tumor pathology. Washington, D.C.

2. Ellis G (2005) Basal cell adenocarcinoma. In: Barnes L, Eveson JW, Reichart P, Sidransky D editors. World Health Organization Classification of Tumours: Pathology and Genetics of Head and Neck Tumours. Lyon, France: IARC Press.

3. de Araújo VC (2005) Basal cell adenoma. In: Barnes L, Eveson JW, Reichart P, Sidransky D editors. World Health Organization Classification of Tumours: Pathology and Genetics of Head and Neck Tumours. Lyon, France: IARC Press 2005.

4. El-Naggar AK, Huvos AG (2005) Adenoid cystic adenocarcinoma. In: Barnes L, Eveson JW, Reichart P, Sidransky D editors. World Health Organization Classification of Tumours: Pathology and Genetics of Head and Neck Tumours. Lyon, France: IARC Press 2005.

5. Mima T, Shirasuna K, Kishino M, Matsuya T (1996) Basal cell adenocarcinoma of the sublingual gland: report of a case. J Oral Maxillofac Surg 54: 1121-1123.

6. Tse GM, To EW, Yuen EH, Chen M (2001) Basal cell adenocarcinoma of the salivary gland: report of a case with morphology on fine needle aspiration cytology. Acta Cytol 45: 775-778.

7. Mardi K, Kaushal V, Asotra S (2011) Basal cell adenocarcinoma of submandibular salivary gland-problems in cytologic diagnosis. below J Cytol 28: 203-206.

8. Jung MJ, Roh JL, Choi SH, Nam SY, Kim SY, et al. (2013) Basal cell adenocarcinoma of the salivary gland: a morphological and immunohistochemical comparison with basal cell adenoma with and without capsular invasion. Diagn Pathol 8: 171. 